\title{
A preliminary Investigation of Surface Micro Modification Effects on the Biocompatibility of 316L Stainless Steel
}

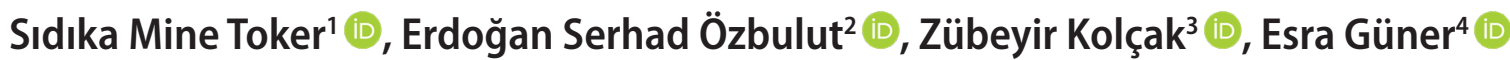 \\ 1-4Eskişehir Osmangazi University (ESOGU), Metallurgical and Materials Engineering Department, Eskişehir, Turkey
}

\begin{abstract}
Effects of surface properties such as roughness and surface energy are critical for determining the biocompatibility of all types of biomaterials, as in the case of biomedical alloys. Recently, microstructure induced surface energy changes have started attracting attention in surface property related biocompatibility analysis of metals. The current study mainly aims to focus on the effects of surface micro modification on the biocompatibility of metallic biomaterials, in order to get an understanding of the underlying mechanisms that affect surface properties and biocompatibility. For this purpose, a preliminary biocompatibility analysis was conducted on a conventional biomedical alloy; 316L stainless steel, whose surface was modified by forming micro-deformation areas of different patterns. The results of this study indicated that, in addition to surface roughness, microdeformation pattern characteristics are also very critical parameters in terms of determining cellular response.
\end{abstract}

Keywords: Biomedical alloys, microstructure, biocompatibility, surface, micro-deformation.

\section{INTRODUCTION}

Metallic biomaterials are widely used for biomedical purposes such as orthopedic implants, dental applications or cardiovascular devices. The main reason biomedical alloys are preferred for these applications despite the development of lighter and advanced materials is their mechanical properties $[1,2]$. Especially for orthopedic applications such as hip and knee implants, the mechanical durability and the ability to easily tailor mechanical properties of metals through altering their microstructure provide an important advantage for metallic biomaterials over other alternatives $[1,2]$. However, due to issues such as potential corrosion, toxic ion release, bacterial layer formation as well as mechanical mismatch with surrounding tissue or insufficient biomechanical fixation, their biocompatibility is yet to be improved [1-4].

For biocompatibility, as in all types of materials, material surface is of utmost importance for metals as well, as the first biomaterial-tissue interaction takes place at the surface $[5,6]$. Surface properties such as roughness, topographical features, wettability and surface energy are the important surface related parameters which determine initial biocompatibility response in terms of biomechanical fixation, osseointegration, corrosion and ion release [5-7]. Among these parameters, the effect of surface roughness is more commonly investigated in relation with surface energy $[8,9]$. As opposed to the general understanding that increased surface roughness is expected to also increase cellular attachment due to increased surface energy, recent studies have shown that organization of the topographical features on the surface may more effectively determine cell response [8-12]

Moreover, some of the recent studies have started exploring the effect of microstructural properties of metallic materials on their biocompatibility [7, 10-12]. Commonly, effects of microstructural mechanisms on the material properties of metallic materials are usually explored with a focus on mechanical properties and deformation behavior. However, recent studies have shown that, microstructural properties of metallic materials can also be critical for determining their biocompatibility at different levels [7, 10-12]. For instance, ex situ biocompatibility was observed to be influenced by the presence of dislocations through localization of oxide particles around dislocation networks, which may affect ion release [7]. Surface topography and surface energy were also shown to be affected by microstructure at the micro and nanoscale, which in turn influenced cell attachment and proliferation behavior [10-12].

With this motivation, the current study aims to get a preliminary understanding of the effect of surface micro modification on the biocompatibility of metallic biomaterials. For this purpose, a conventional biomedical alloy; 316L stainless steel was investigated by forming controlled micro-defor-
* Corresponding author

Email: stoker@ogu.edu.tr
European Mechanical Science (2021), 5(3): 109-115

doi: https://doi.org/10.26701/ems.820904

Received: November 4, 2020

Accepted: March 17, 2021 
mation areas on the sample surfaces and the effect of each micro-deformation area on cell attachment was examined.

\section{MATERIALS AND METHODS}

The 316L steel samples, for which the chemical composition is given in Table 1 , were cut in pieces of $3 \mathrm{~mm}$ height from a $5 \mathrm{~mm}$ radius cylindrical rod. The surfaces of each sample were then prepared for surface micro deformation via grinding and polishing.

Controlled micro-deformation areas with different patterns of micro-indentations were formed on $316 \mathrm{~L}$ stainless steel samples by using a Vickers micro hardness testing device. Details of the formed micro-deformation areas in terms of the applied load, formed indent size and the length of the spacings between indent centers are given in Table 2. A control sample without any micro-deformation areas was also investigated for comparison, which is referred to as the 'reference' sample. The obtained surfaces were examined with
Hitachi Regulus 8230 Field Emission Scanning Electron Microscope (FESEM) and surface roughness measurements were performed with a Mitutoyo mechanical contact needle profilometer.

Table 2. Properties of the micro-deformation areas formed on $316 \mathrm{~L}$ sample surface.

\begin{tabular}{|c|c|c|c|}
\hline Sample & $\begin{array}{c}\text { Applied load and expo- } \\
\text { sure period }\end{array}$ & $\begin{array}{c}\text { Average } \\
\text { indent size } \\
(\mu \mathrm{m})\end{array}$ & $\begin{array}{c}\text { Average spacing } \\
\text { between indent cen- } \\
\text { ters }(\mu \mathrm{m})\end{array}$ \\
\hline Reference & - & - & - \\
\hline 1 & $1 \mathrm{KgF}, 5$ Seconds & 80 & 100 \\
\hline 2 & $1 \mathrm{KgF}, 5$ Seconds & 80 & 200 \\
\hline 3 & $0.5 \mathrm{KgF}, 5$ Seconds & 60 & 100 \\
\hline 4 & $0.5 \mathrm{KgF}, 5$ Seconds & 60 & 200 \\
\hline
\end{tabular}

For the in vitro tests, Saos-2 cells (osteosarcoma cell line) were seeded on the reference sample surface and on the the micro-deformation areas of the patterned samples with a density of 5000 cells per sample. Cells were fixated on the sample surfaces with the use of a glutaraldehyde agent, fol-

Table 1. Chemical composition of the investigated $316 \mathrm{~L}$ stainless steel in weight percent.

\begin{tabular}{|c|c|c|c|c|c|c|c|c|c|c|c|}
\hline Element & $\mathrm{Fe}$ & $\mathrm{C}$ & $\mathrm{Mn}$ & $\mathrm{P}$ & $\mathrm{S}$ & $\mathrm{Cr}$ & $\mathrm{Ni}$ & $\mathrm{Mo}$ & $\mathrm{Cu}$ & $\mathrm{Si}$ & $\mathrm{N}$ \\
\hline $\begin{array}{c}\text { Weight per- } \\
\text { cent (\%) }\end{array}$ & Balance & 0,027 & 1,76 & 0,042 & 0,029 & 16,80 & 10,12 & 2,12 & 0,38 & 0,27 & 0,08 \\
\hline
\end{tabular}

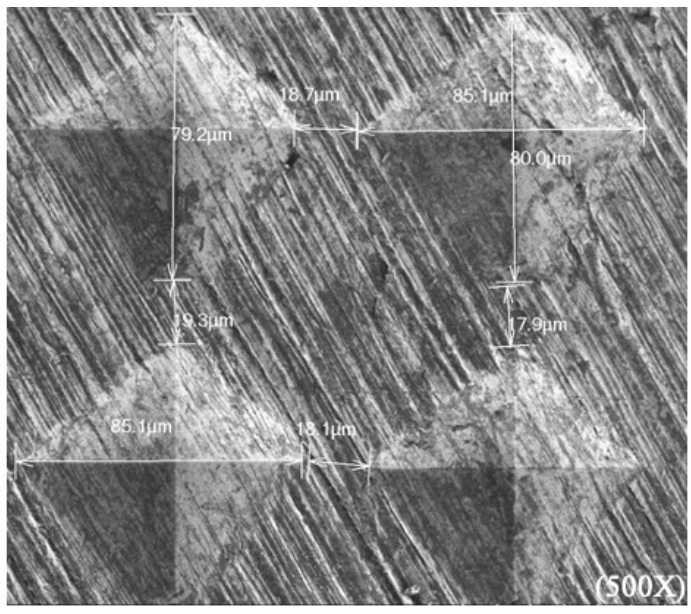

(a)

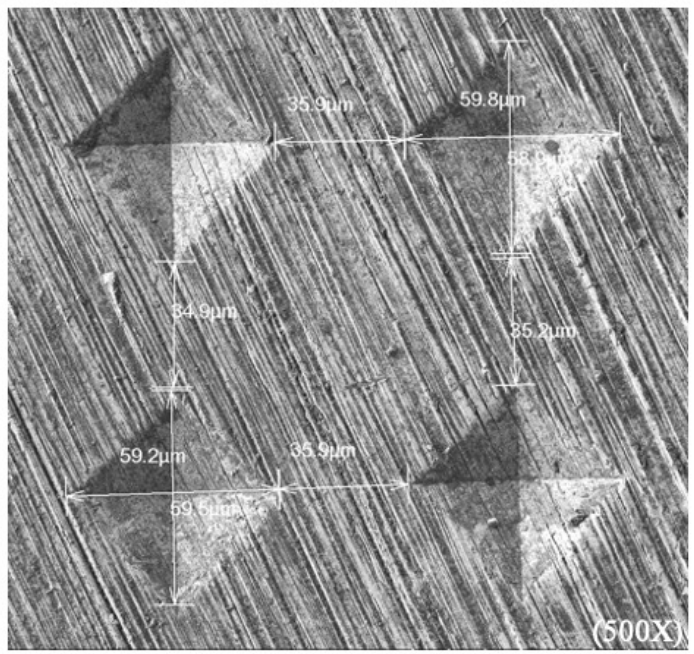

(c)

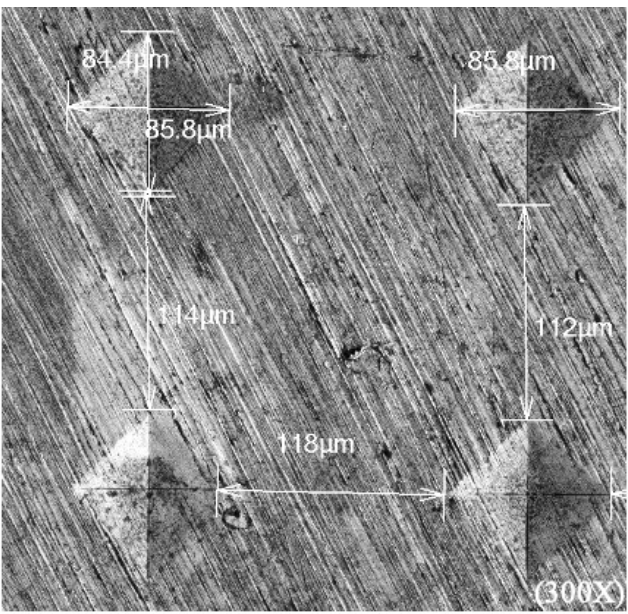

(b)

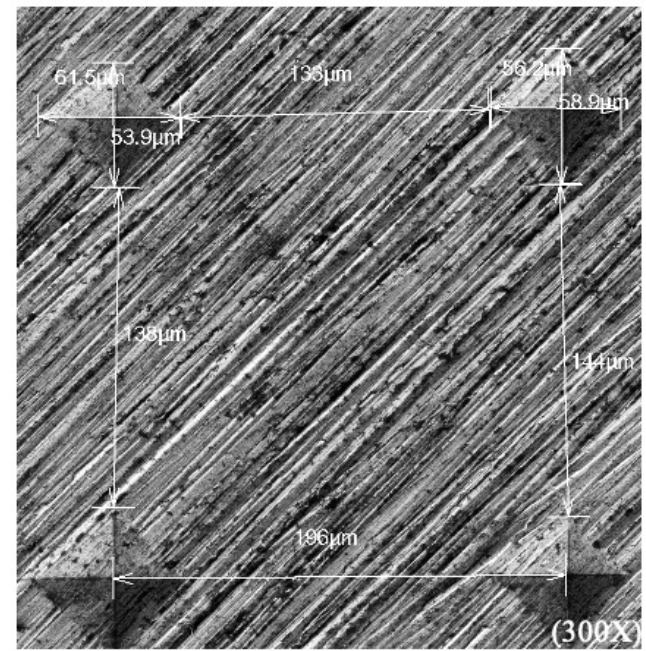

(d)

Figure 1. FESEM images from the initial microstructural characterization of the micro-deformation areas of; (a) Sample-1, (b) Sample-2, (c) Sample-3, (d) Sample-4. 
lowing incubation periods of 5 and 10 days. Cell morphologies were then examined in detail via FESEM. With the use of the FESEM images, cell count on each micro-deformation area was performed and converted to number of cells per millimeter square $\left(\mathrm{mm}^{2}\right)$ area for comparison.

\section{RESULTS AND DISCUSSION}

Initial structural characterizations of the formed micro-deformation areas of the four patterned sample surfaces were performed via FESEM (Figure 1). These initial examinations evidenced that the indentation size and spacings were formed as planned and no unexpected structural irregularities were observed within or around the micro-deformation areas (Figure 1).

The results of the surface profilometry analysis of the formed patterns and the reference sample surface listing the average surface roughness $(\mathrm{Ra})$ and the maximum depth $(\mathrm{Rz})$ created by the indentations for each pattern are given in Table 3. (Since no indentations were formed on the reference sample, Rz value was not measured for this sample.)

Table 3. Results of surface profilometry analysis

\begin{tabular}{|c|c|c|}
\hline Sample & Average $\mathrm{Ra}(\mu \mathrm{m})$ & Maximum $\mathrm{Rz}(\mu \mathrm{m})$ \\
\hline Reference & 0.32 & - \\
\hline 1 & 1.19 & 9.96 \\
\hline 2 & 0.99 & 9.74 \\
\hline 3 & 0.79 & 7.37 \\
\hline 4 & 0.49 & 5.81 \\
\hline
\end{tabular}

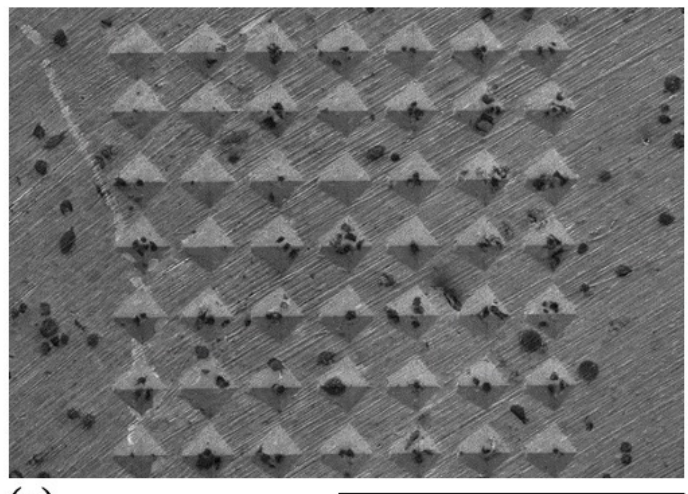

(a)

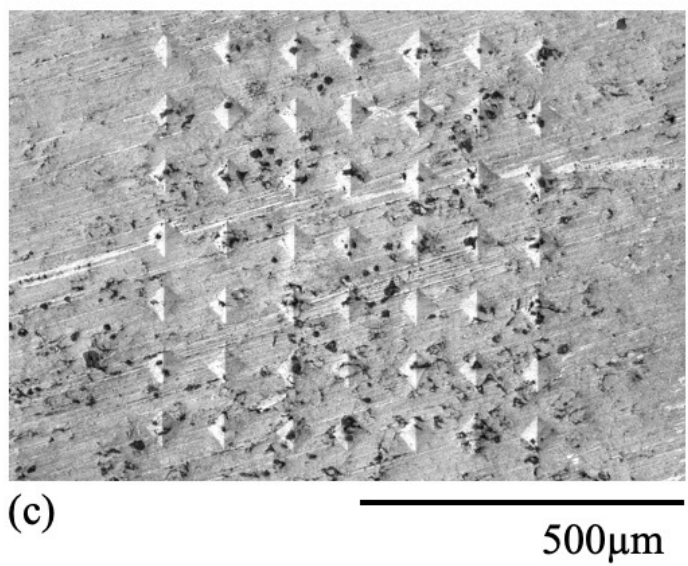

The surface profilometry analysis results revealed all of the micro-deformation areas exhibit much higher average roughness values as compared to the reference sample. Moreover, comparing the deformed sample surfaces with each other, it was observed that average surface roughness increases with increasing indent size and the application of a larger amount of load results in deeper indents which translate to higher $\mathrm{Rz}$ values as observed in samples 1 and 2 in comparison to samples 3 and 4. On the other hand, comparing the effect of spacing length between the indentations, it can be argued that roughness increases as the space between indentation centers decrease, which can be observed for samples 1 and 3 as compared to samples 2 and 4 .

In order to get an understanding of the effects of varying surface properties on cell attachment behavior as a function of time, the Saos-2 cells fixated on each sample were observed in detail via FESEM following incubation periods of 5 and 10 days. The cells localized on the patterned area were investigated at different magnifications, where the overall cellular behavior on the whole micro-deformation area (Figure 2 and Figure 4) and on individual indents (Figure 3 and Figure 5) were examined in detail. The cell attachment behavior on the reference sample surfaces following incubation periods of 5 and 10 days were also investigated and are given in Figure 6.

Comparing the micro-deformation areas to the reference surface, it was observed that all the samples with surface

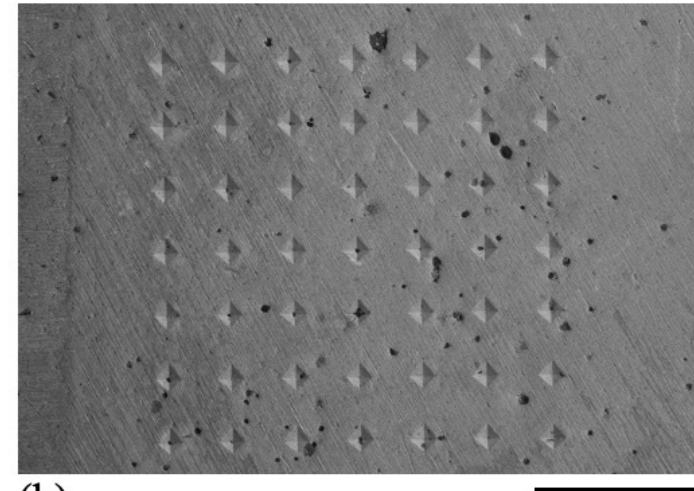

(b)

$500 \mu \mathrm{m}$

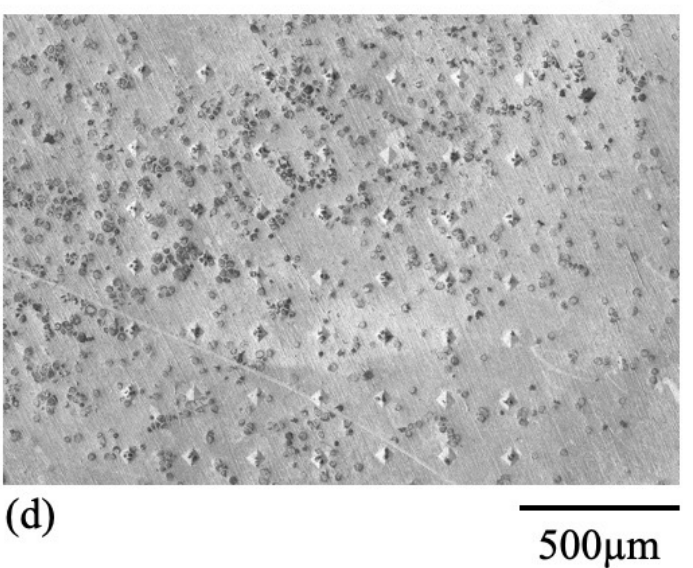

Figure 2. FESEM images of the Saos-2 cells fixated on the overall micro-deformation areas of (a) Sample-1, (b) Sample-2, (c) Sample-3, (d) Sample-4; following 5 days of incubation. 


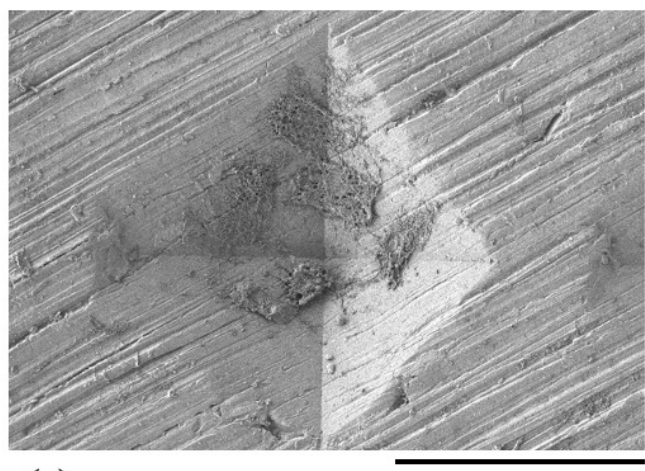

(a)

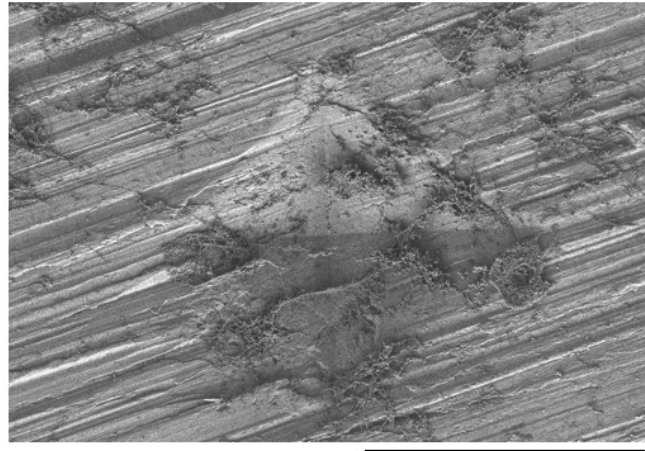

(c)

$50 \mu \mathrm{m}$
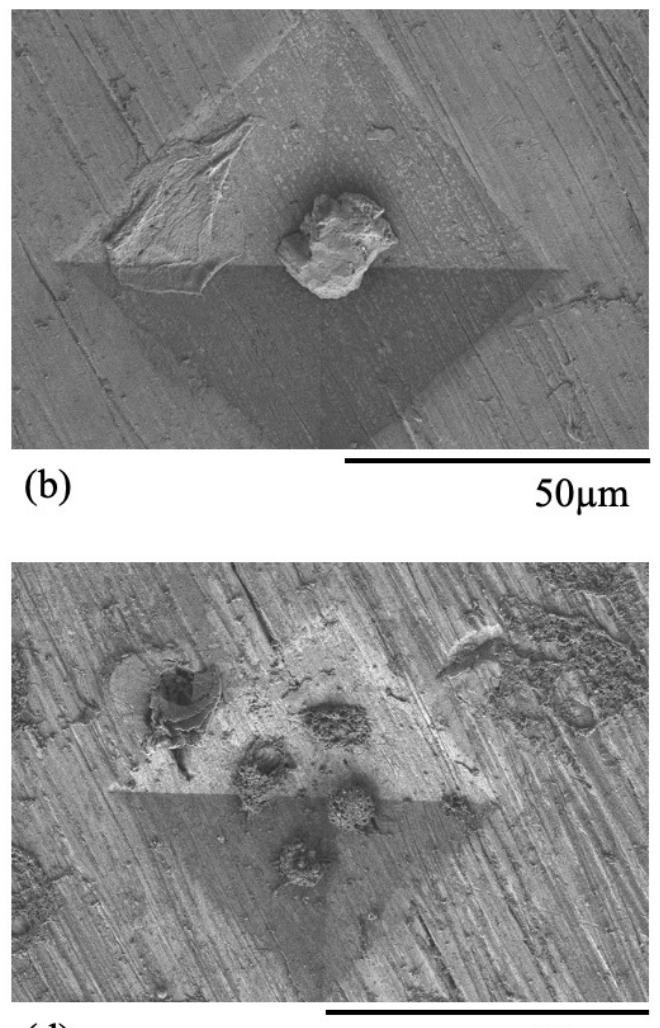

(d)

$50 \mu \mathrm{m}$

Figure 3. FESEM images of the Saos-2 cells around individual indents of (a) Sample-1, (b) Sample-2, (c) Sample-3, (d) Sample-4; following 5 days of incubation.

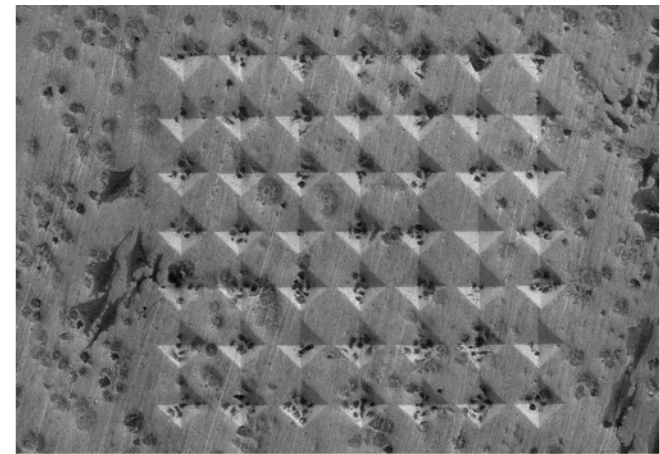

(a)

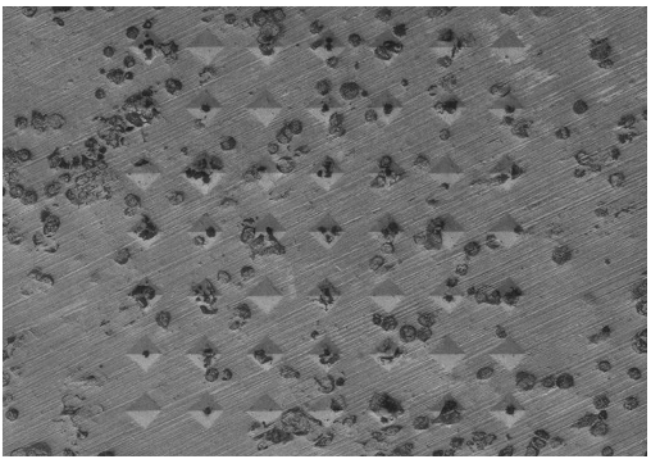

(c)

$500 \mu \mathrm{m}$
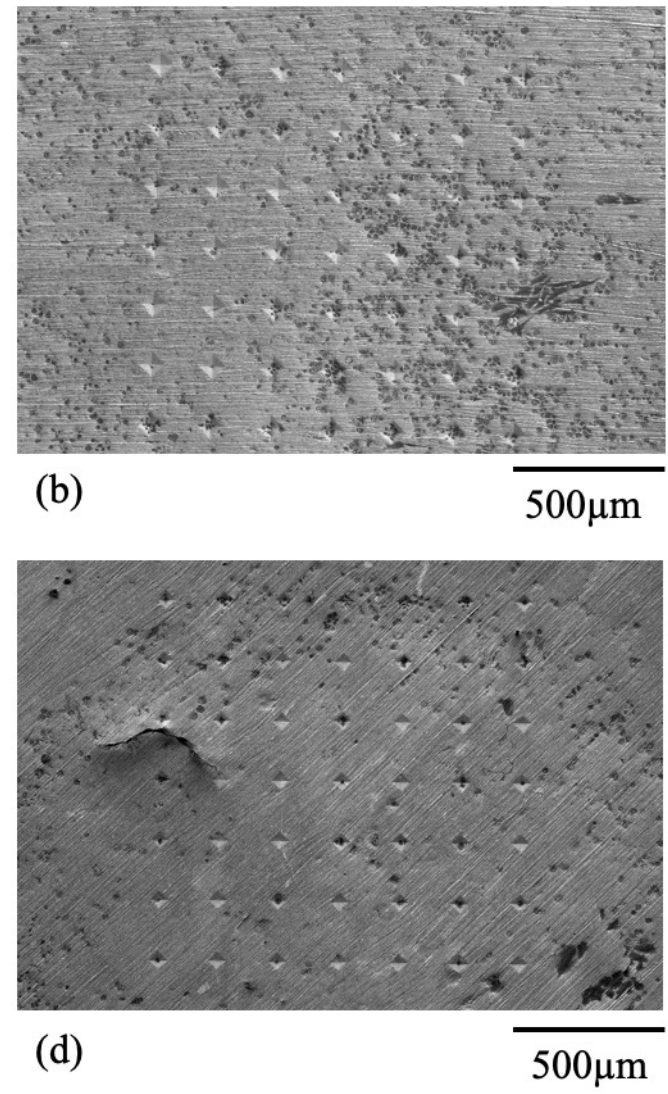

Figure 4. FESEM images of the Saos-2 cells fixated on the overall micro-deformation areas of (a) Sample-1, (b) Sample-2, (c) Sample-3, (d) Sample-4 following 10 days of incubation.

processing exhibited higher cell density on their micro-deformation areas as compared to the reference sample on the $5^{\text {th }}$ day of incubation, suggesting a positive effect stemming from micro-deformation (Figure 6 (a)). On the other hand, attachment behaviors of the Saos-2 cells fixated on patterned samples exhibited significant differences depending 


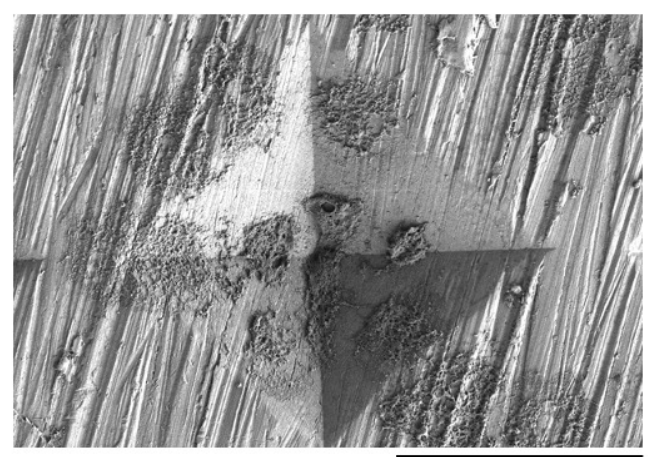

(a)

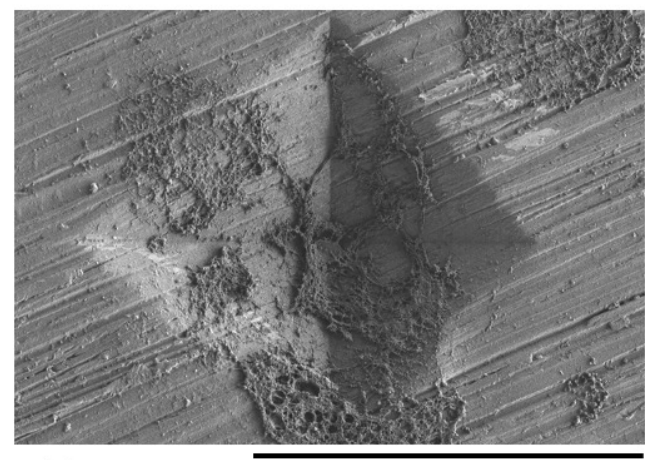

(c)
$50 \mu \mathrm{m}$

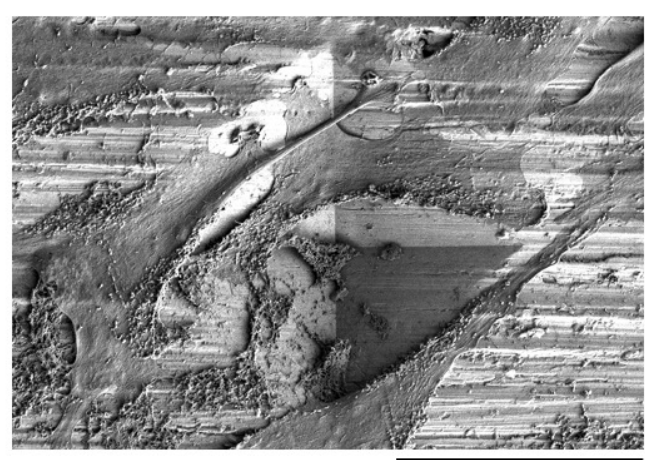

(b)

$50 \mu \mathrm{m}$

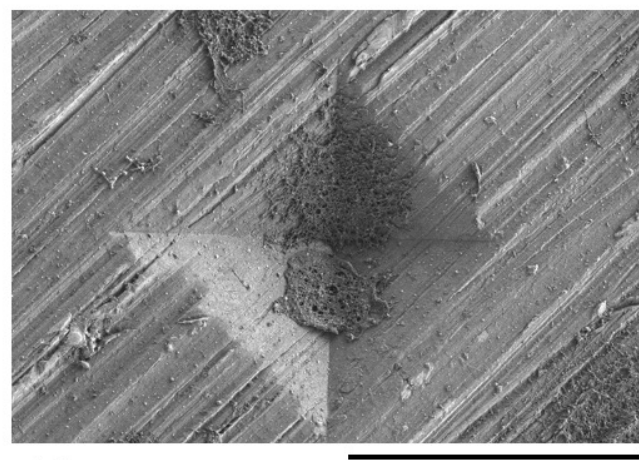

(d)

$50 \mu \mathrm{m}$

Figure 5. FESEM images of the Saos-2 cells around individual indents of (a) Sample-1, (b) Sample-2, (c) Sample-3, (d) Sample-4; following 10 days of incubation.

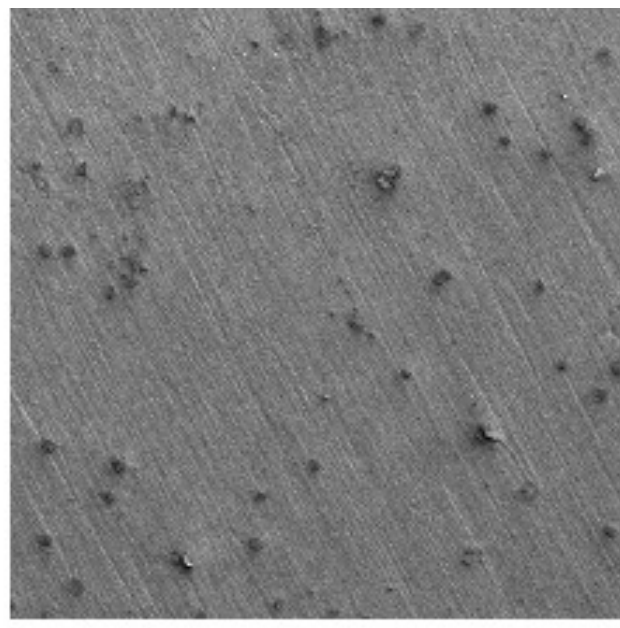

(a)

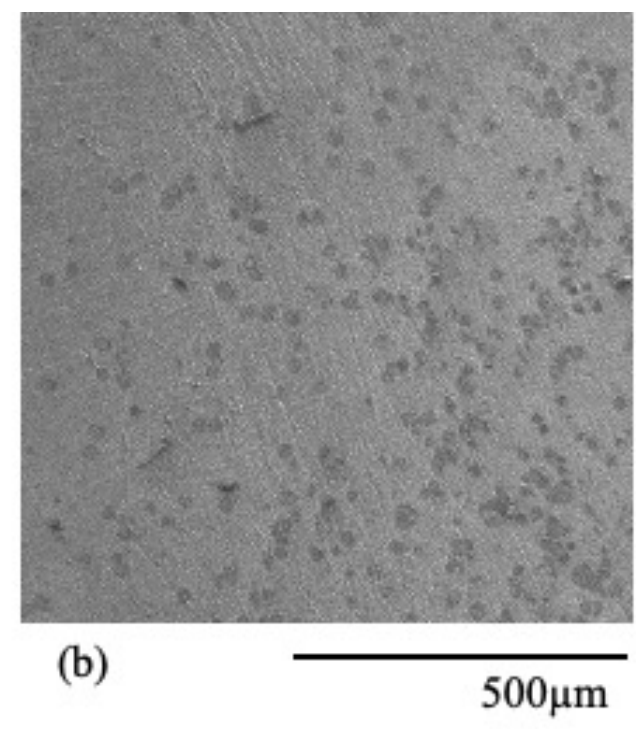

Figure 6. FESEM images of the Saos-2 cells on the reference sample surface (a) following 5 days of incubation, (b) following 10 days of incubation.

on the micro-deformation pattern and varying roughness values. (Figures 2-5). Specifically, according to Figure 2, on the $5^{\text {th }}$ day of incubation; a higher number of cells were apparent on samples 3 and 4, which exhibit lower surface roughness and shallower indents as compared to samples 1 and 2. The closer look on individual indents also yielded a similar result with higher cell density located within and around the indents of samples 3 and 4. These results showed that, up to 5 days of incubation, increased surface roughness had a negative effect on cell attachment for the samples with micro-deformation.

However, on the $10^{\text {th }}$ day of incubation, a completely dif- ferent cellular behavior was observed. The general view of the patterned areas exhibited higher numbers of cells on samples 1 and 2 this time, as compared to samples 3 and 4 (Figure 4). Moreover, higher densities of cells were apparent within and around individual indents of samples 1 and 2 as well, in comparison to samples 3 and 4 (Figure 5). These findings indicated that increased surface roughness exhibited a positive effect on cell attachment on the $10^{\text {th }}$ day of incubation, contradicting the observations from day 5 of incubation. Therefore, it can be argued that, in addition to surface roughness, another parameter affecting this behavior is indent characteristics. Specifically, the larger indent size of 
samples 1 and 2 enabled localization of a higher number of cells over time, which also contributed to increased cellular attachment on these surfaces through $10^{\text {th }}$ day of incubation. On the other hand, the cell density on the surface of the reference sample appeared to be lower as compared to the patterned surfaces, demonstrating the positive effect of surface micro-deformation on cell attachment behavior on the $10^{\text {th }}$ day of incubation as well.

In order to make a quantitative evaluation in addition to visual observations, the number of cells on the patterned area of each sample on each incubation period were counted and presented as a bar graph comparing the numbers of cells per $\mathrm{mm}^{2}$ area (Figure 7). These quantitative results support the observations from the FESEM images as well. Specifically, this graph also reveals an inverse correlation between surface roughness and cell attachment at the early stage of incubation, whereas at the later stage of incubation, increased surface roughness appears to promote cellular attachment and proliferation for the surfaces with micro-deformation. Also, the higher numbers of cells per $\mathrm{mm}^{2}$ on all of the micro-deformation surfaces in comparison to the reference sample surface, support the FESEM observations where surface-micro-deformation processing in general appear to promote cellular attachment.

Moreover, this graph provides a basis for comparing the samples with the same indent size (namely sample 1 vs. 2 and sample 3 vs. 4) with each other. According to this comparison, initially at both incubation periods, the numbers of cells were higher on sample 1 than sample 2. Sample 1 exhibited the highest surface roughness and in many studies higher surface roughness was observed to positively influence cellular attachment due to the increased surface energy $[8,9]$. However, comparing the percentage change in the number of cells over time, the number of cells increased by $174 \%$ on sample 1 , while a significant increase of $380 \%$ was apparent on sample 2. This observation is an important indicator of how a direct correlation may not always be present between surface roughness and cell attachment. The cell count comparisons from the $5^{\text {th }}$ day of incubation also evidence a similar finding where surfaces with lower roughness values resulted in higher numbers of cell attachment. On the other hand, from the comparison between samples 3 and 4, whose indent sizes are the same, it can be observed that this time the sample with the higher surface roughness, namely sample 3, appears to promote cellular attachment and proliferation more than sample 4, with similar percentage changes over time. Lastly, comparing samples 1 and 3 with samples 2 and 4 in order to get an understanding of the effect of indent spacing; it can be argued that closer indent spacing also exhibits a more positive effect on cell attachment.

Overall, these results indicate that, in addition to surface roughness, the indent size and the spacing are also important parameters affecting the surface energy and thus cell attachment behavior on the surface. Moreover, the surface energy changes stemming from micro-structural mecha- nisms which are induced by the formation of these micro indents, are also expected to be effective in the observed results of cell attachment and proliferation behavior. Similar results where the effects of the structural features and micro-structural mechanisms were more prominent in terms of affecting cellular behavior as compared to surface roughness were reported in literature as well [10-12]. Therefore, it can be argued that surface energy changes stemming from microstructural mechanisms constitute another important factor affecting cellular response and should be investigated in detail in the future studies.

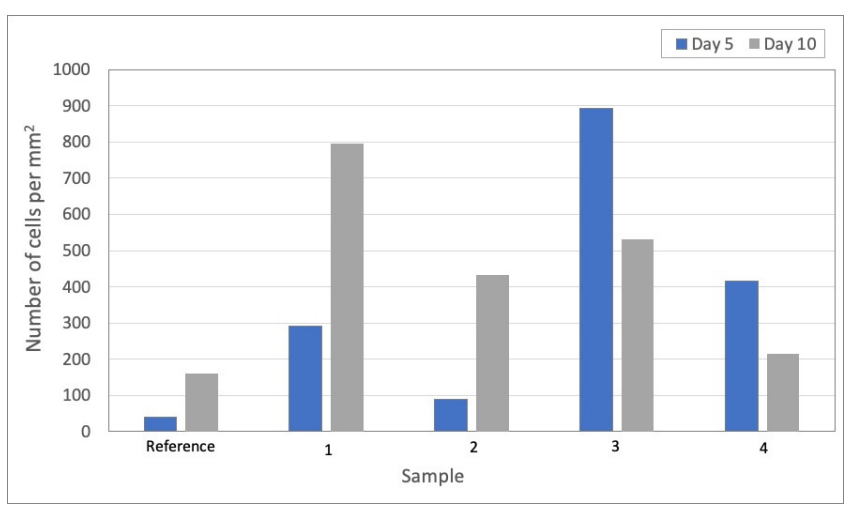

Figure 7. The number of cells per $\mathrm{mm}^{2}$ area on the reference sample surface and micro-deformation area of each sample on the $5^{\text {th }}$ and $10^{\text {th }}$ days of incubation.

\section{CONCLUSION}

The findings of this study revealed that cell attachment and proliferation behaviors were not only influenced by surface roughness, but also by the specifics of the surface features of the different micro-deformation areas. Specifically, the indent size and spacings were also found to play a determining role on the cell attachment and proliferation behavior. Moreover, these results suggest that the effects of micro-structural mechanisms on surface energy, which were induced by micro-deformation, can also influence the cellular behavior and this effect should be investigated in detail in the future studies.

\section{ACKNOWLEDGEMENTS}

This work was financially supported by the ESOGU Bilimsel Araştırma Projeleri (BAP) Grant, Project \# 2018/15038. The authors would also like to thank the members of ESOGU Cellular Therapy and Stem Cell Production, Application and Research Center (ESTEM) for their help through the in vitro experiments.

\section{REFERENCES}

[1] Bauer, S., Schmuki, P., Mark, K.V.D., Park, J. (2013). Engineering Biocompatible Implant Surfaces Part I: Materials and Surfaces. Progress in Materials Science, 58(3):261-326, DOl: 10.1016/j.pmatsci.2012.09.001.

[2] Manam, N.S., Harun, W.S.W., Shri, D.N.A., Ghani, S.A.C., Kurniawan, T., Ismail, M.H., Ibrahim, M.H.I. (2017). Study of Corrosion in Biocompatible Metals for Implants: A Review. Journal of Alloys and Compounds, 701: 698-715, DOI: 10.1016/j.jallcom.2017.01.196.

[3] Toker, S.M., Canadinc, D., Maier, H.J., Birer, O. (2014). Evaluation of 
Passive Oxide Layer Formation- Biocompatibility Relationship in NiTi Shape Memory Alloys: Geometry and Body Location Dependency. Materials Science and Engineering C, 36:118-129, DOI: doi. org/10.1016/j.msec.2013.11.040.

[4] Lu, T., Wen, J., Qian, S., Cao, H., Ning, C., Pan, X., Jiang, X., Liu, X., Chu, P. K. (2015). Enhanced Osteointegration on Tantalum- Implanted Polyetheretherketone Surface with Bone-Like Elastic Modulus. Biomaterials, 51: 173-183, DOI: 10.1016/j.biomaterials.2015.02.018.

[5] Keyf, F., Uzun, G. (2007). Surface Characteristics of the Implant Systems and Osseointegration. Journal of Atatürk University Faculty of Dentistry, 2: 43-50.

[6] Sahin, C., Korkmaz, C., Uzun, G. (2015). Osseointegration, Surface Porosity and Nanotechnology. Journal of Atatürk University Faculty of Dentistry, 25(13): 174-181, DOI: 10.17567/dfd.90462.

[7] Toker, S.M., Gerstein, G., Maier, H.J., Canadinc, D. (2018). Effects of Microstructural Mechanisms on The Localized Oxidation Behavior of NiTi Shape Memory Alloys in Simulated Body fluid. Journal of Materials Science, 53: 948-958, DOl:10.1007/s10853-017-1586-4.

[8] Venkatsurya, P.K.C., Thein, W.W., Misra, R.D.K., Somani, M.C., Karjalainen, L.P. (2010). Advancing Nanograined/Ultrafine-grained Structures for Metal Implant Technology: Interplay Between Grooving of Nano/Ultrafine Grains and Cellular Response. Materials Science and Engineering C, 30(7):1050-1059, DOl: 10.1016/j. msec.2010.05.008.

[9] Liang, C., Wang, H., Yang, J., Li B., Yang, Y., Li, H. (2012). Biocompatibility of The Micro-Patterned NiTi Surface Produced by Femtosecond Laser. Applied Surface Science, 261: 337-342, DOI: 10.1016/j. apsusc.2012.08.011.

[10] Uzer, B., Toker, S.M., Cingoz, A., Bagci-Onder, T., Gerstein, G., Maier, H.J., Canadinc, D. (2016). An Exploration of Plastic Deformation Dependence of Cell Viability and Adhesion in Metallic Implant Materials. Journal of the Mechanical Behavior of Biomedical Materials, 60: 177-186. DOI: 10.1016/j.jmbbm.2016.01.001.

[11] Toker, S.M., Sugerman, G., Frey, E.C. (2019). Effects of Surface Characteristics on the in vitro Biocompatibility Response of NiTi Shape Memory Alloys. Academic Platform Journal of Engineering and Science, 7(2): 112-116. DOI: 10.21541/apjes.461169.

[12] Uzer, B. (2020) Modulating the Surface Properties of Metallic Implants and the Response of Breast Cancer Cells by Surface Relief Induced via Bulk Plastic Deformation. Frontiers in Materials,7: 1-10. DOI:10.3389/fmats.2020.00099. 